\title{
The effect of docosahexaenoic acid on predicting the survival of patients with idiopathic pulmonary arterial hypertension
}

\author{
Xu Huai ${ }^{1,2 \#}$, Yuanyuan Sun ${ }^{1 \#}$, Xiuying Sun ${ }^{3 \#}$, Wenhui Wu ${ }^{1}$, Lan Wang ${ }^{1}$, Rong Jiang ${ }^{1}$, Sugang Gong ${ }^{1}$, \\ Jinling $\mathrm{Li}^{1}$, Yuqing Miao ${ }^{2}$, Ping Yuan ${ }^{1}$, Qinhua Zhao ${ }^{1}$ \\ ${ }^{1}$ Department of Cardio-Pulmonary Circulation, Shanghai Pulmonary Hospital, Tongji University, School of Medicine, Shanghai, China; ${ }^{2}$ Institute \\ of Bismuth Science, University of Shanghai for Science and Technology, Shanghai, China; ${ }^{3}$ Department of Fever, Weifang Traditional Chinese \\ Hospital, Weifang, China \\ Contributions: (I) Conception and design: P Yuan, Q Zhao, X Huai, Y Sun, X Sun; (II) Administrative support: None; (III) Provision of study \\ materials or patients: None; (IV) Collection and assembly of data: X Huai, Y Sun, X Sun, W Wu, R Jiang, L Wang, J Li, P Yuan; (V) Data analysis \\ and interpretation: X Huai, Y Sun, X Sun, P Yuan; (VI) Manuscript writing: All authors; (VII) Final approval of manuscript: All authors. \\ \#These authors contributed equally to this work. \\ Correspondence to: Qinhua Zhao, MD; Ping Yuan, MD. Department of Cardio-Pulmonary Circulation, Shanghai Pulmonary Hospital, Tongji \\ University, School of Medicine, No. 507 Zhengmin Road, Shanghai 200433, China. Email: zhaoqinhua@tongji.edu.cn; pandyyuan@tongji.edu.cn; \\ Yuqing Miao, MD. Institute of Bismuth Science, University of Shanghai for Science and Technology, Shanghai 200093, China. \\ Email: yqmiao@ymail.com.
}

Background: Abnormal lipid metabolism has been reported in patients with idiopathic pulmonary arterial hypertension (IPAH); however, the prognostic value of plasma free fatty acids (FFAs) for these patients is unclear. The present study aimed to determine whether FFA can play a role in predicting the survival of patients with IPAH.

Methods: A total of 69 blood samples from patients with IPAH were subjected to liquid chromatographymass spectrometry (LC-MS). According to the classification criteria for pulmonary hypertension in the European Society of Cardiology (ESC) guidelines, patients were divided into low-risk, intermediate-risk, and high-risk groups. The FFA expression levels of patients in the three groups were compared, and the indicators with significant differences were selected. Cox regression analysis was performed to examine the associations between survival and different factors. Receiver operator characteristic (ROC) curves were used to assess the predictive effect of plasma lipids in assessing patients' risk of morbidity, including area under the curve (AUC), sensitivity, specificity and the best cutoff value. Kaplan-Meier curves were used to predict survival.

Results: A total of 24 FFA molecules were detected in the patients with IPAH. Among them, FFA (20:4), FFA (20:5), FFA (22:5), FFA (22:6), FFA (24:0) and FFA (30:4) showed significant differences between the low-risk and the intermediate-risk or high-risk patients with IPAH. These six FFAs were significantly correlated with hemodynamic parameters. FFA (22:6), named docosahexaenoic acid (DHA), displayed significant negative correlations with World Health Organization functional classification (WHO FC), mean right atrial pressure (mRAP), and pulmonary vascular resistance (PVR), and significant positive correlations with 6-minute walking distance (6MWD) and cardiac index (CI). Cox regression analyses demonstrated that total bilirubin (TBIL) and DHA were independent risk factors for survival of IPAH. Receiver operating characteristic curve analysis revealed that DHA had a cut-off value of 77.55 , which had a sensitivity of $96.7 \%$ and a specificity of $62.5 \%$ for predicting survival. Kaplan-Meier curve analysis showed that a lower level of DHA predicted a poor outcome in patients with IPAH.

Conclusions: Our study suggested that FFA levels were correlated with disease severity. Lower levels of DHA predict poor survival in patients with IPAH.

Keywords: Idiopathic pulmonary arterial hypertension (IPAH); free fatty acids (FFAs); docosahexaenoic acid (DHA); prognosis; biomarker 
Submitted Apr 29, 2021. Accepted for publication Jun 15, 2021.

doi: 10.21037/atm-21-2479

View this article at: https://dx.doi.org/10.21037/atm-21-2479

\section{Introduction}

Idiopathic pulmonary arterial hypertension (IPAH) is a rare but devastating disease characterized by vascular remodeling in the pulmonary artery wall, which leads to increased pulmonary vascular resistance (PVR), right ventricular hypertrophy, and, ultimately, right heart failure (1). The incidence rate of IPAH is about $6 / 1$ million (2), and the fatality rate is high, the mean time from symptom onset to diagnosis was 2 years, the median survival time was 2.8 years, and the 1-, 3- and 5-year survival rates were $68 \%, 48 \%$, and $34 \%$, respectively $(3,4)$. Due to the lack of specific clinical symptoms and signs of IPAH, it is very easy to be misdiagnosed. When the disease is diagnosed, it often has progressed to the medium-term or more serious stage and has poor prognosis, so their quality of life is not significantly improved after treatment (5). Moreover, the pathophysiological mechanism of IPAH is complex, the pulmonary vasculature is dynamically obstructed by vasoconstriction. Vascular cells (endothelial cells, smooth muscle cells, and fibroblasts) and inflammatory cells are abnormal proliferation and resistance to apoptosis or aggregation in IPAH. Genetic, epigenetic dysregulation, the autoimmune, inflammatory disease, ischemia and fibrosis of the right ventricle and so on are also contributes to disease pathogenesis (1). Therefore, the specific etiology of IPAH has not been thoroughly and clearly explained, and the changes of clinical indicators and the potential related mechanisms still need to be explored in IPAH in order to find an accurate and specific diagnostic marker using a lowcost and safe method which will improve the prognosis of patients with the disease.

With the gradual maturity and improvement of lipidomics technology in recent years, lipid metabolism has been widely studied (6). Extensive research of the pathways and networks of lipid metabolism has shown that changes in lipid levels can reflect variations at several enzymatic levels, activities, and/or gene expression patterns. Lipids are important biomolecules which are widely distributed and abundant in living organisms, they have diversified structures, so they have a variety of important functions in various biological progresses. Not only they are the important components of cells and organelles, but also because they take part in energy conversion, material transport, information recognition, signal transmission, cell growth, differentiation and apoptosis of cells (7). Therefore, lipid metabolism and its functional changes have a critical impact on the physiological functions of cells and pathological disorders in living organisms. Abnormal lipid metabolism is frequently closely related to metabolic diseases, cardiovascular diseases, and tumors (8). Dysregulation of lipid metabolism can also occur in IPAH, such as abnormally elevated triglycerides and HDL, which often indicate inflammation (9).

Free fatty acids (FFAs) are the intermediate products of lipid metabolism and the important metabolic substrates of cell energy metabolism in the body, which provides energy for the metabolism of the body. Some basic studies have proved that some lipid such as FFAs have a positive protective effect on the cardiovascular system, among which docosahexaenoic acid (DHA) mediated pulmonary artery hyperpolarization through $\mathrm{KCa}$ channels can lead to rapid pulmonary artery dilation, which theoretically has a certain relieving effect on the symptoms of patients with pulmonary hypertension (10). However, they did not asses the expressions and potential effects of plasma DHA in patients.

In the present study, we systematically explored the differences about FFAs expression in patients with IPAH using liquid chromatography-mass spectrometry (LC-MS) for the first time and evaluated the use of FFAs in predicting the survival of these patients.

We present the following article in accordance with the STARD reporting checklist (available at https://dx.doi. org/10.21037/atm-21-2479).

\section{Methods}

\section{Sample selection and blood collection}

A total of 69 incident patients with IPAH who were treated at Shanghai Pulmonary Hospital between May 2013 and April 2019 were enrolled in this retrospective study. In accordance with the European Society of Cardiology (ESC) guidelines, a diagnosis of IPAH was defined as mean pulmonary arterial pressure $(\mathrm{mPAP}) \geq 25 \mathrm{mmHg}$, pulmonary 
capillary wedge pressure (PCWP) $\leq 15 \mathrm{mmHg}$, and PVR $>3$ Wood units, as measured by right heart catheterization (RHC) $(11,12)$. Patients associated with connective tissue disease, congenital heart disease, portal hypertension, pulmonary disease, chronic thromboembolism, left heart disease, or anorexigens were excluded. Patients with acute or chronic diseases that might affect hormone metabolism, including acute or chronic infections, chronic autoimmune diseases, and previously identified primary endocrine disorders were also excluded, as were those who were receiving or who had previously received hormones (thyroid hormones, anabolic steroids, or corticosteroids) or medications that significantly inhibit hormone production.

This study complied with the Declaration of Helsinki (as revised in 2013) and was approved and supervised by the ethics committee of Shanghai Pulmonary Hospital (number: K20-195Y), and written informed consent was obtained from all study participants.

\section{Sample processing}

Each plasma sample $(50 \mu \mathrm{L})$ was put in a glass tube, and $750 \mu \mathrm{L} \mathrm{MeOH}$ containing $50 \mu \mathrm{L}$ of internal standard (corticosterone-d8, $10 \mu \mathrm{g} / \mathrm{mL}$ ) was added. After vortexing for 2 minutes, $2.5 \mathrm{~mL}$ dichloromethane (DCM) was added to the mixture and vortexing was performed for a further 10 minutes. Then, $625 \mu \mathrm{L}$ deionized water was added, and vortexing was performed again, followed by centrifugation for 15 minutes at 3,000 rpm to separate the mixture into different liquid phase stratification. The lower layer was collected and transferred to a new glass tube. The upper layer was secondarily extracted with $2 \mathrm{~mL}$ DCM. Finally, the pooled organic layer was combined and evaporated until dry. The dried extract was reconstituted in $200 \mu \mathrm{L}$ of Ammonium acetate: $\mathrm{H}_{2} \mathrm{O}(1: 1, \mathrm{~V} / \mathrm{V})$ for LC-MS/MS analysis. Quality control (QC) samples were prepared by mixing an equal volume of all samples, and the same preparation procedure was followed.

\section{LC-MS detection}

The supernatant was analyzed by hydrophilic interaction chromatography-high performance liquid chromatography and tandem mass spectrometry (HILIC-HPLC-MS/MS) using a TripleQUAD 6500 mass spectrometer (AB SCIEX, USA) coupled with an ExionLC liquid chromatography system (AB SCIEX, USA). For LC separation, the BEH amide HILIC column $(100 \mathrm{~mm} \times 2.1 \mathrm{~mm}$ i.d., $1.7 \mu \mathrm{m}$;
Waters) was used. A $5 \mu \mathrm{L}$ sample was injected and separated using a 16-minute gradient. The column flow rate was maintained at $500 \mu \mathrm{L} /$ minute, with a column temperature of $40^{\circ} \mathrm{C}$.

The electrospray ionization mass spectra were acquired in positive ion mode and negative ion mode, respectively. The transition information of target lipids was collected using the multi-reaction monitor (MRM) mode. The ion spray voltage was set to 5,500 and $4,500 \mathrm{~V}$ for positive mode and negative mode, respectively; the declustering potential (DP) was set to $80 \mathrm{~V}$; and the heated capillary temperature was maintained at $550{ }^{\circ} \mathrm{C}$. The curtain gas flow, nebulizer, and heater gas were set to 35,55 , and 55 arbitrary units, respectively.

\section{Clinical data collection}

Data of the patients were collected including: demographic information; World Health Organization functional class (WHO FC); 6-minute walking distance (6MWD); $\mathrm{N}$-terminal fragment of pro-brain natriuretic peptide (NT-proBNP); hemodynamic parameters, including mean right atrial pressure (mRAP), mPAP, mean pulmonary artery wedge pressure (mPAWP), PVR, cardiac index (CI), mixed venous oxygen saturation $\left(\mathrm{SvO}_{2}\right)$; other laboratory parameters; and treatment. A patient's risk level can be determined based on the following six indicators: WHO FC, 6MWD, mRAP, CI, $\mathrm{SvO}_{2}$, and NT-proBNP (13). According to ESC guideline, 69 patients with IPAH were divided into low-risk, intermediate-risk, and high-risk groups for subsequent statistical analyses. The all-cause mortality rate was estimated from the confirmation date of IPAH patients to December 2020.

\section{Statistical analysis}

Data were expressed as means with standard deviations or medians with interquartile ranges for continuous variables, and as numbers and percentages for categorical variables. Hazard ratios (HRs) were calculated along with $95 \%$ confidence intervals (95\% CIs). The statistical software IBM SPSS Statistics (IBM, USA) 24 was used for the analysis of all data, including clinical physiological parameters and the relative quantity of lipid molecules in all patients. Patients were divided into the low-risk, intermediate-risk, and highrisk groups according to the classification criteria set out in the European Disease Guidelines in order to evaluate the relationship between the established classification index 
parameters and lipid molecules, as potential biomarkers of IPAH.

Statistical analysis strategy: first, one-way analysis of variance (ANOVA) and the Kruskal-Wallis test were used to determine whether significant differences existed in the relative quantity levels of lipid molecules among different risk groups. Pearson's correlation analysis was performed between the relative quantity of lipid molecules detected and the physiological parameters of mature diseases to investigate the predictive trend of these potential biomarkers. Further, univariate and multivariate Cox regression analyses were carried out for the selected indicators after the two analyses to further screen for lipids that have a good predictive effect on mortality in patients with different risk levels. Then, receiver operator characteristic (ROC) curve analysis was performed for the re-screened indicators that had a significant effect on IPAH disease risk. And the cutoff value that resulted in the highest product of sensitivity and specificity was considered the optimal threshold for prognostication. Finally, Kaplan-Meier survival curve analysis was carried out. The correlation between factors and risk of disease was visualized. $\mathrm{P}<0.05$ was considered statistically significant

\section{Results}

\section{Baseline characteristics}

Table 1 displays the clinical characteristics, hemodynamics laboratory results, and specific therapy of the study participants at baseline. The mean age of the 69 patients with IPAH was $33.3 \pm 12.0$ years; $80.3 \%$ patients were female; and 38 (62.3\%) patients were WHO group III/ IV. According to ESC guideline, 69 IPAH patients were divided into 3 groups for subsequent statistical analysis, with 28,25 , and 16 patients classified as low, intermediate, and high risk, respectively. During an average follow-up of 69 [8-92] months, 8 patients (11.59\%) died.

No significant difference was found among the different groups in terms of age, mPAP, or mPAWP. However, there were significant differences with respect to sex, WHO FC, 6MWD, NT-proBNP, mRAP, PVR, CI, and $\mathrm{SvO}_{2}$. Patients in the present study received treatments including targeted medications including phosphodiesterase type 5 inhibitors (sildenail, tadalafil, and vadenafil), oral endothelial receptor antagonists (ambrisentan and bosentan), and soluble guanylate cyclase stimulator (riociguat), as well as combination therapy and nonspecific medications.
There were no apparent differences relating to the use of medications between the different risk groups.

\section{FFA detection and comparison among different groups with IPAH}

In this study, MultiQuan (Sciex ${ }^{\mathrm{TM}}$ ) software was used for peak extraction, peak matching, peak alignment, and normalization of the original sample quality spectrum data. Based on the self-built lipid standard spectrographic library, the identification list was generated by extracting lipid substances with a signal-to-noise ratio $>3$, and the peak area was normalized on the basis of internal standard substances. In this detection process, unsupervised principal component analysis (PCA) was used to observe the overall distribution between samples and the degree of dispersion between groups (Figure 1A). Then supervised orthogonal partial least squares discrimination analysis (OPLS-DA) was used to distinguish the overall differences in metabolic profiles between groups and to look for the metabolites that differed between groups (Figure 1B). The response intensity of 24 FFA molecules was regarded as effective (Figure 1C).

One-way ANOVA was used to test whether for significant differences in the relative quantity of FFAs between the different risk groups. The results are shown in Table 2. FFA (20:4), FFA (20:5), FFA (22:5), FFA (22:6), FFA (24:0), and FFA (30:4) showed significant differences in expression, which was consistent with the FFA subclass change trend, and the levels in the low-risk group were significantly higher than those in the intermediate- and high-risk groups. As shown in Figure 2A. FFA (22:6), known as DHA, showed the most significant difference between the low-risk and high-risk groups. Further, the levels of DHA in the low-risk group were higher than those in the intermediate-risk group. Surviving patients had higher DHA levels than non-surviving patients (Figure 2B). No significant sex differences were found in FFA (20:4), FFA (20:5), FFA (22:5), DHA, FFA (24:0), or FFA (30:4) levels among patients with IPAH (Figure 2C).

\section{Correlations of FFAs with clinical parameters in patients with IPAH}

Spearman's correlation analysis was conducted between several FFAs and baseline clinical parameters of the patients including WHO-FC, NT-proBNP, mRAP, mPAP, mPAWP, CI, $\mathrm{SvO}_{2}$, and PVR. The results obtained are shown in Figure 3. FFA (20:4), FFA (20:5), FFA (22:5), DHA, FFA 
Table 1 Characteristics of the study population

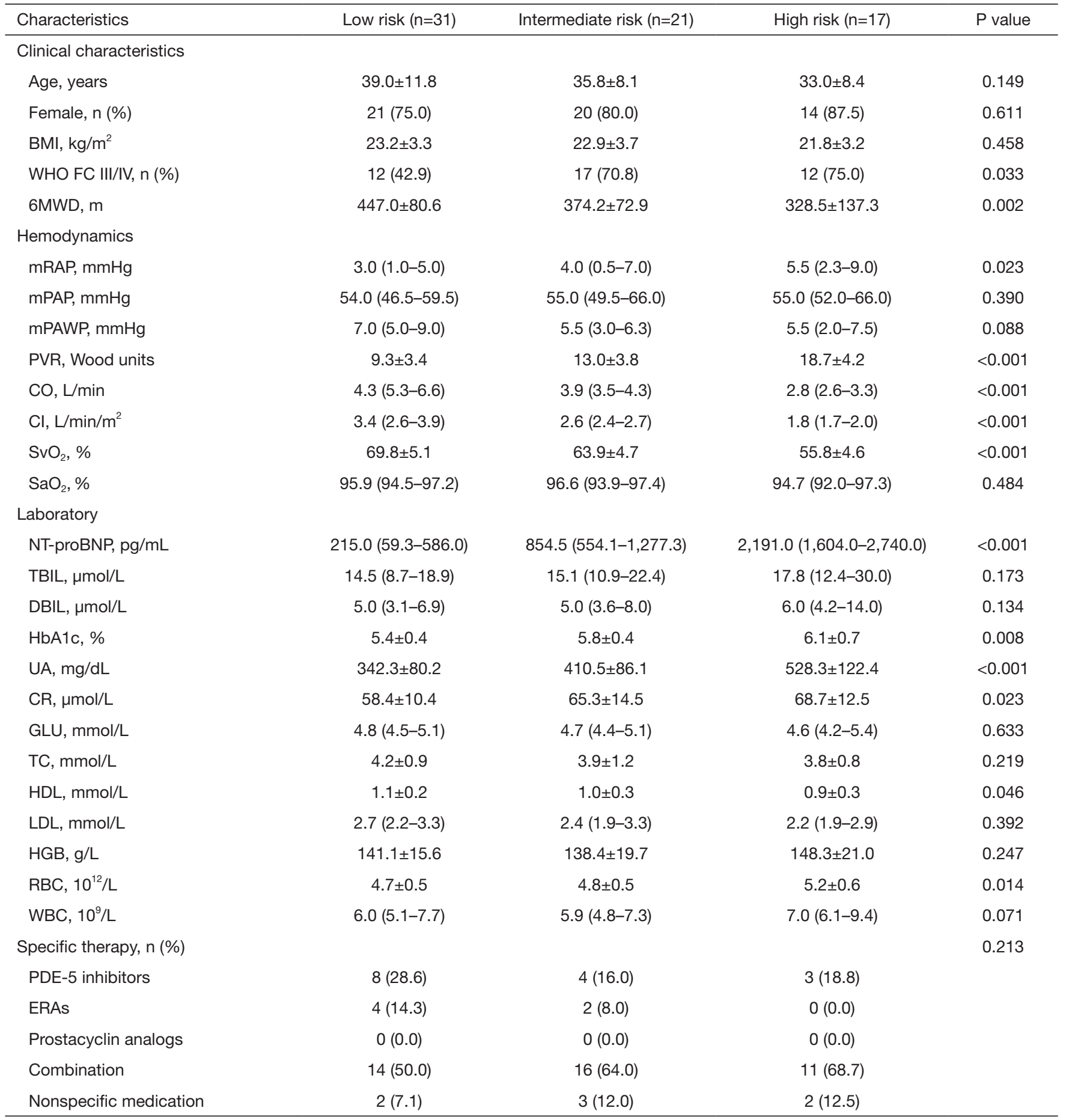

Data are presented as $\mathrm{n}(\%)$, mean $\pm \mathrm{SD}$, and interquartile range. BMI, body mass index; WHO FC, World Health Organization functional classification; 6MWD, 6-minute walking distance; mRAP, mean right atrial pressure; mPAP, mean pulmonary arterial pressure; mPAWP, mean pulmonary artery wedge pressure; PVR, pulmonary vascular resistance; $\mathrm{CO}$, cardiac output; $\mathrm{Cl}$, cardiac index; $\mathrm{SvO}_{2}$, mixed venous oxygen saturation; $\mathrm{SaO}_{2}$, mixed arterial oxygen saturation; NT-proBNP, N-terminal pro-brain natriuretic peptide; TBIL, total bilirubin; DBIL, direct bilirubin; HbA1c, hemoglobin A1c; UA, uric acid; CR, creatinine; GLU, glucose; TC, total cholesterol; HDL, high-density lipoprotein; LDL, low-density lipoprotein; HGB, hemoglobin; RBC, red blood cell; WBC, white blood cell; PDE-5, phosphodiesterase 5; ERA, endothelin receptor antagonist. 
A

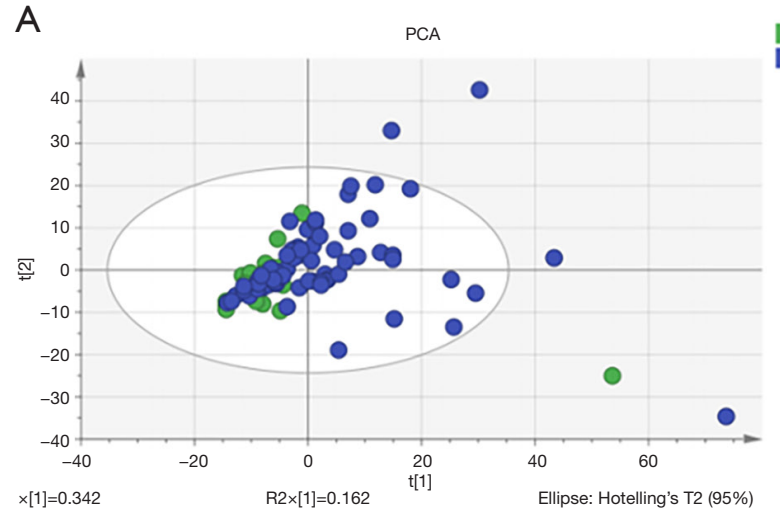

$\times[1]=0.342$

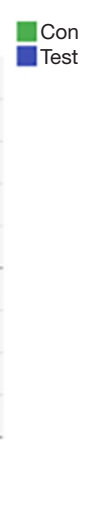

B

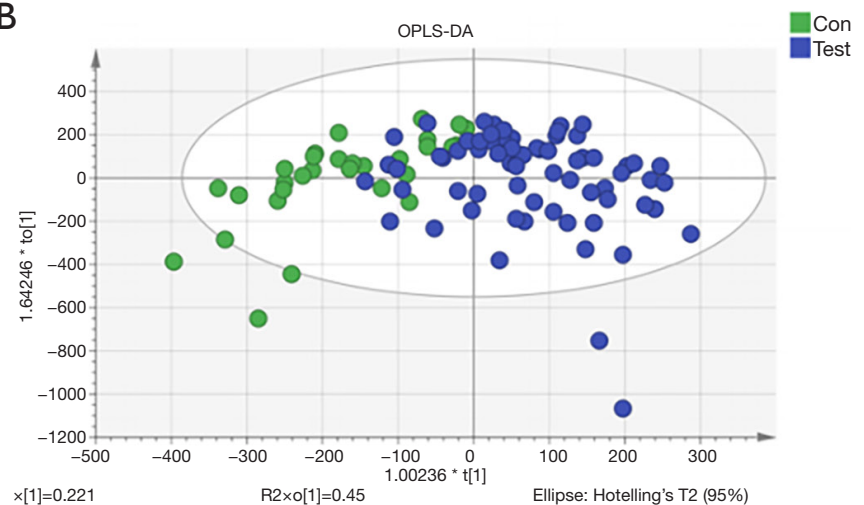

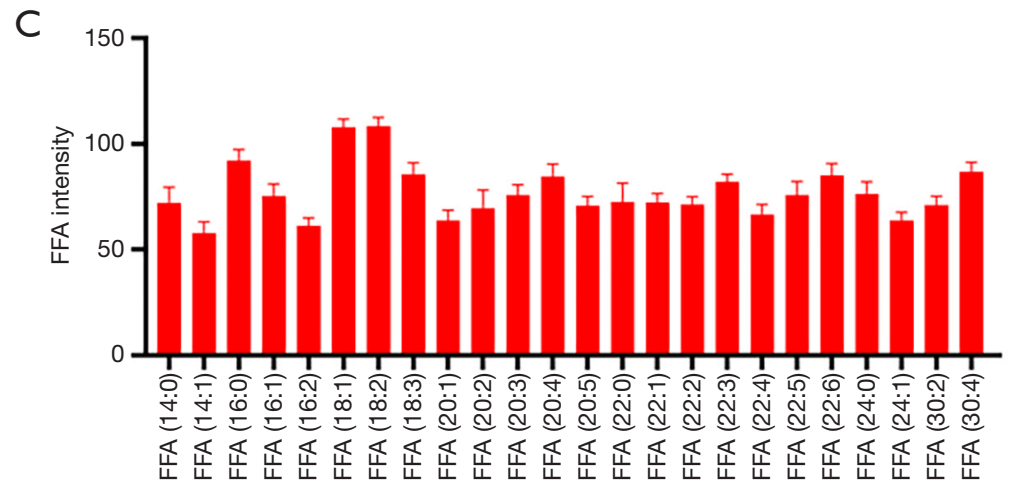

Figure 1 Initial data processing for LC-MS. (A) Plasma lipids of each sample were analyzed by PCA; (B) plasma lipids of each sample were analyzed by OPLS-DA; (C) statistical diagram of FFAs quantities. The types and total number of FFAs detected in this experiment. LC-MS, liquid chromatography-mass spectrometry; PCA, principal component analysis; OPLS-DA, orthogonal partial least squares discrimination analysis; FFA, free fatty acid.

(24:0), and FFA (30:4) were significantly correlated with several clinical parameters. In particular, the 6 FFAs were all significantly negatively correlated with NT-proBNP, and positively correlated with CI. WHO-FC was significantly negatively correlated with FFA (20:5), FFA (22:5), DHA, FFA (24:0), and FFA (30:4). PVR was significantly negatively correlated with FFA (20:4), FFA (20:5), FFA (22:5), DHA, and FFA (24:0). Also, mRAP was significantly negatively correlated with FFA (20:4), FFA (20:5), FFA (22:5), and DHA. However, mPAWP showed no significant correlation with any of the six FFAs. Furthermore, DHA had significant negative correlations with WHO-FC, mPAP, PVR, and NT-proBNP, but significant positive correlations with 6MWD and CI.

\section{Cox regression analysis of FFAs in patients with IPAH}

To further explore the impact of the above-mentioned factors on patient survival, we conducted univariate and multivariate Cox regression analyses (Figure 4). In the univariate analysis, parameters such including high-density lipoprotein (HDL), total bilirubin (TBIL), creatinine (CR), FFA (20:5), and DHA were identified as predictors of survival in patients with IPAH. The subsequent multivariable analysis indicated that DHA was significantly and independently associated with mortality in patients with IPAH.

\section{$D H A$ can predict the survival of patients with IPAH}

ROC analyses were conducted to determine the sensitivity and specificity of DHA as a predictor of mortality in patients with IPAH. The area under the curve (AUC) for DHA was 0.845 , and the cut-off value was 77.55 , which had a sensitivity of 0.967 and a specificity of 0.625 for predicting IPAH survival (Figure 5). According to the cut-off value of DHA, the patients were grouped into the high DHA and low DHA groups. Kaplan-Meier curve analysis revealed 
Table 2 Comparison of FFA levels among different risk groups of patients with IPAH

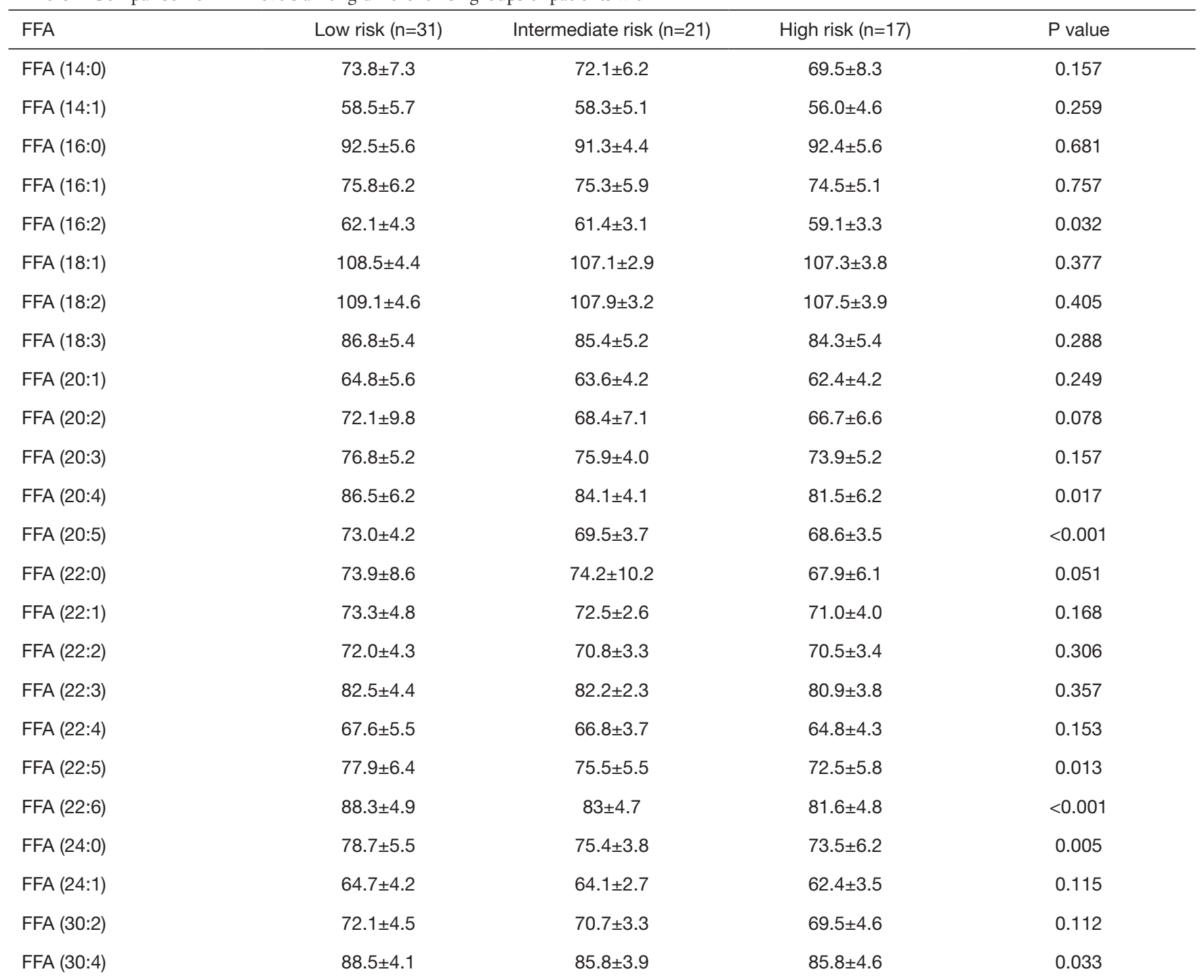

Data in the table are presented in the form of mean \pm standard deviation. FFA (A:B): $A$ is the number of carbon atoms in the molecule; $B$ is the number of unsaturated bonds in the molecule. FFA, free fatty acid; IPAH, idiopathic pulmonary arterial hypertension.

that patients with a high DHA level had better survival than those with a low DHA level (Figure 6).

\section{Discussion}

The present study firstly found that FFA levels were correlated with disease severity. Lower levels of DHA predict poor survival in patients with IPAH. Although the sample number was small, it was basically representative, and the sample source was absolutely reliable. On this basis, the role of lipid metabolism in IPAH was taken as the starting point to conduct lipidomics analysis, to analyze the relationship between FFAs and hemodynamic parameters, and to evaluate the predictive value of FFAs on the survival of patients with IPAH in different risk stratification groups.

In the comparison of FFA levels between patients in different risk groups, the levels of several FFAs in low-risk patients were significantly higher than those in non-low-risk patients, which indicated that these FFAs may be protective factors in patients with IPAH. Furthermore, the data showed that several FFAs were significantly correlated with patients' clinical features and hemodynamic parameters. 
A
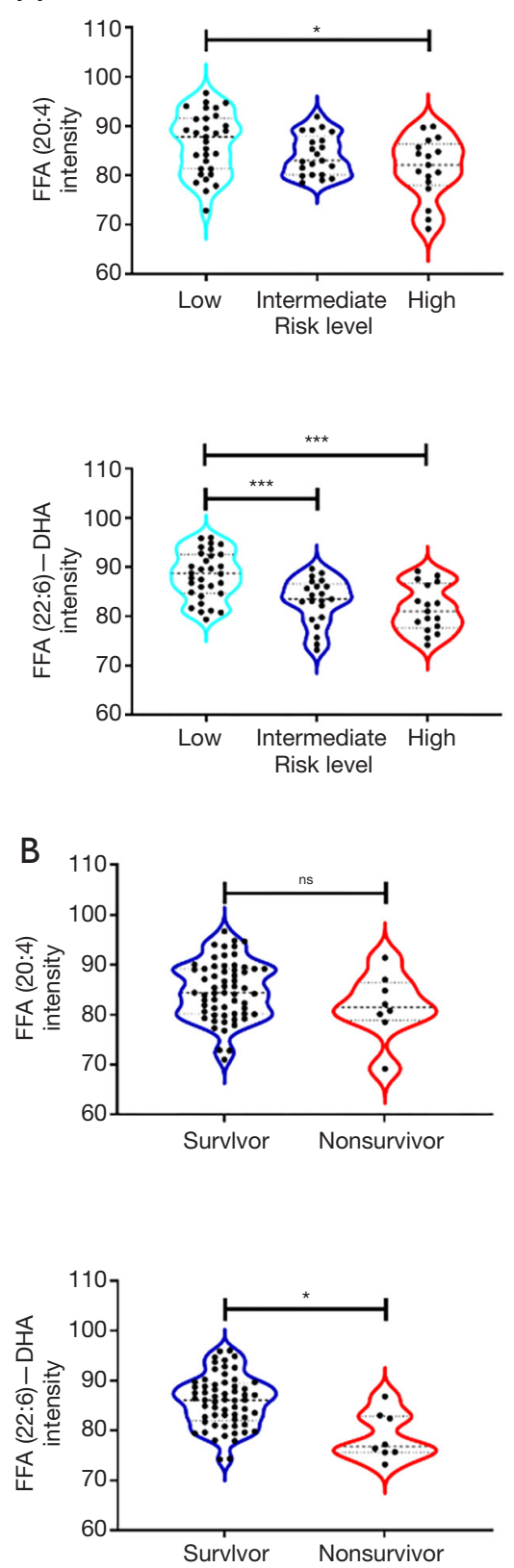
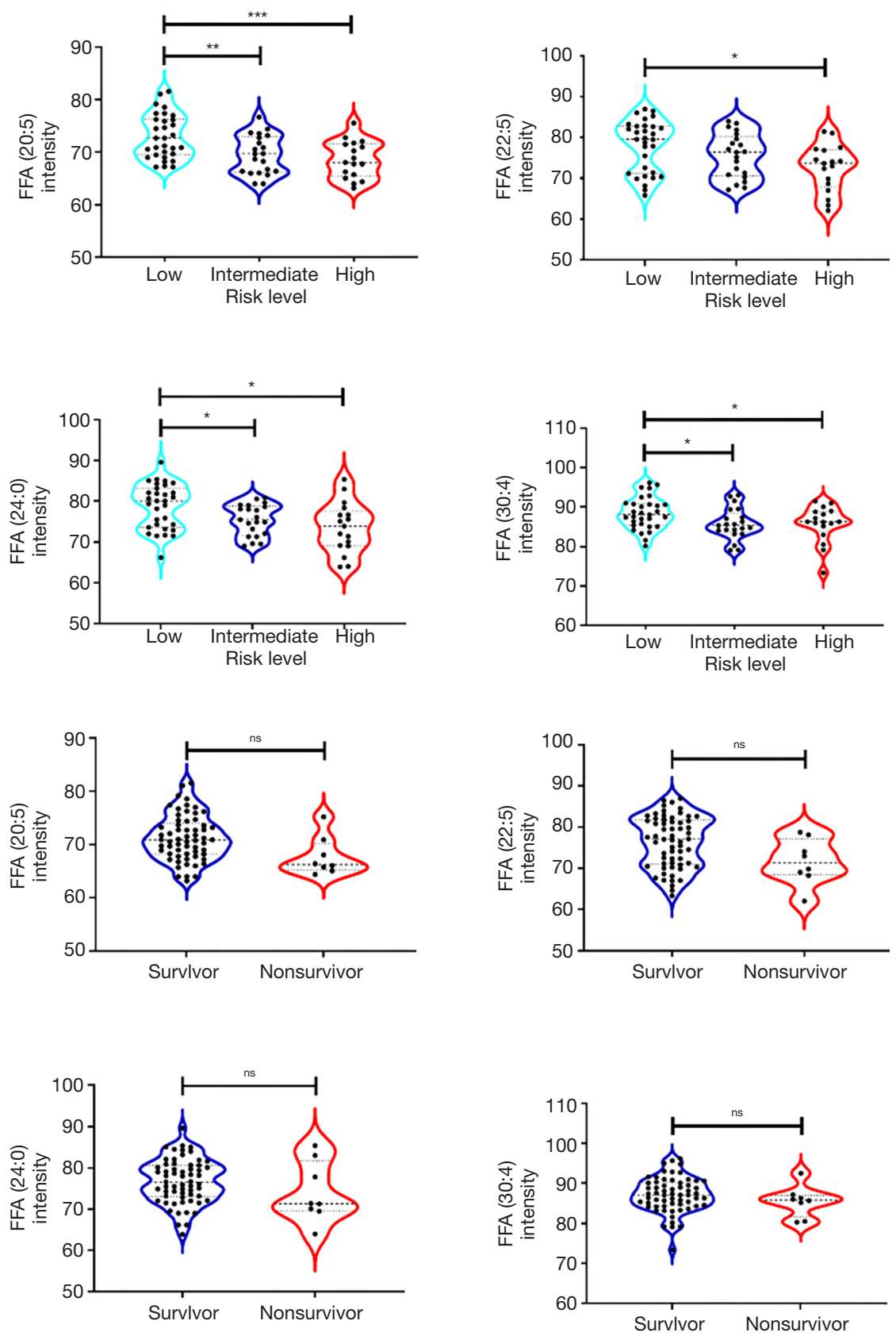

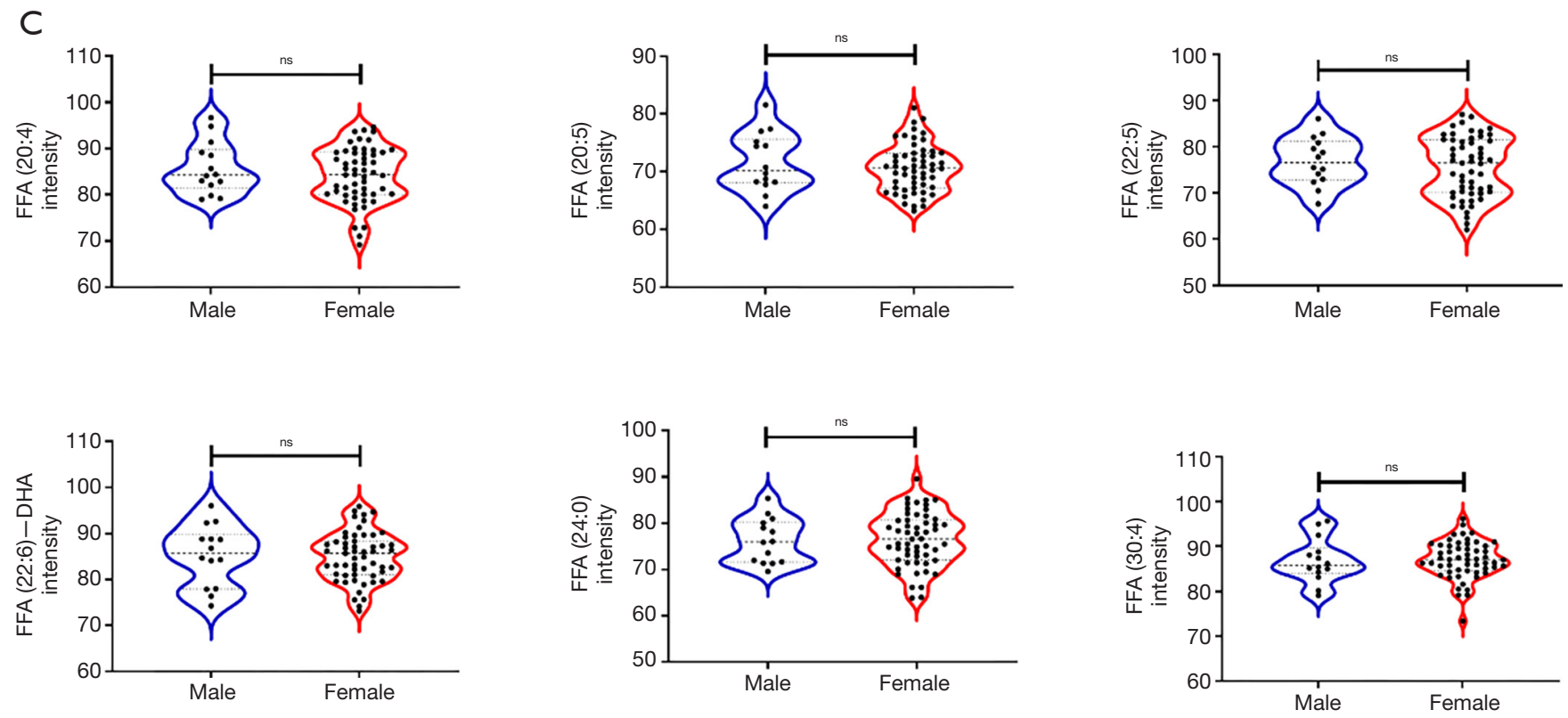

Figure 2 Comparison of FFA levels between different risk groups of patients with IPAH. (A) Comparison of the expression levels of FFAs in the different risk groups; (B) comparison of the expression levels of FFAs in survivor and non-survivors; (C) comparison of the expression levels of FFAs in males and females. *, $\mathrm{P}<0.05$; ${ }^{* *}, \mathrm{P}<0.01$; ${ }^{* * *}, \mathrm{P}<0.001$; ns, $\mathrm{P} \geq 0.05$. FFA, free fatty acid; IPAH, idiopathic pulmonary arterial hypertension.

In particular, PVR was significantly negatively correlated with FFA (20:4), FFA (20:5), FFA (22:5), DHA, and FFA (24:0). Also, mRAP was significantly negatively correlated with FFA (20:4), FFA (20:5), FFA (22:5), and DHA. The results further indicated that the levels of FFAs were related to the development of pulmonary vascular and right heart dysfunction in patients.

Studies have shown that FFAs, also called non-esterified fatty acids, as intermediate products of lipid metabolism in the blood, are important metabolic substrates of cell energy metabolism in the body, providing energy for the metabolism of heart, liver, skeletal muscle, and other organs (14). Many studies have shown that FFAs levels are related to the occurrence and development of metabolic syndrome, atherosclerosis, acute coronary syndrome, heart failure, and other diseases. In the late stage of IPAH development, the right ventricle, which is responsible for pulmonary circulation, will suffer from failure due to the occurrence of thrombus and obstruction of pulmonary artery and the increase of pressure. FFAs are responsible for the energy supply of the right ventricle during this process, and their levels may predict the onset of right heart failure. In addition, FFAs signal transduction is also strongly correlated with pulmonary vasoconstriction, intimal injury and thrombosis $(9,15)$.

FFA (20:5) and FFA (22:6), also known as eicosapentaenoic acid (EPA) and DHA, respectively, were first discovered in an epidemiological study in the late 1960s. The study found that people who ate a diet rich in EPA and DHA had a low incidence of myocardial infarction. Subsequently, a large number of studies were conducted to verify the positive effects of these two FFAs on cardiovascular protection (16). EPA and DHA intake can reduce the occurrence of sudden cardiac death in patients with heart disease events, and can effectively reduce morbidity and mortality among patients with symptomatic chronic heart failure (17). Additionally, many animal studies have shown that small lipid molecules of FFAs, including EPA and DHA, have a variety of beneficial effects in the cardiovascular system, including anti-arrhythmic, antithrombotic, anti-atherosclerotic, endothelial relaxation, blood pressure-lowering, anti-inflammatory, and antifibrotic effects. In terms of pulmonary hypertension, a few studies have also shown that EPA and DHA have antipulmonary hypertension effects, which were mainly realized in a model of hypoxic pulmonary hypertension through relaxation of great artery vessels, reduction of pulmonary vasomotization, and inhibition of pulmonary fibrosis (18). 


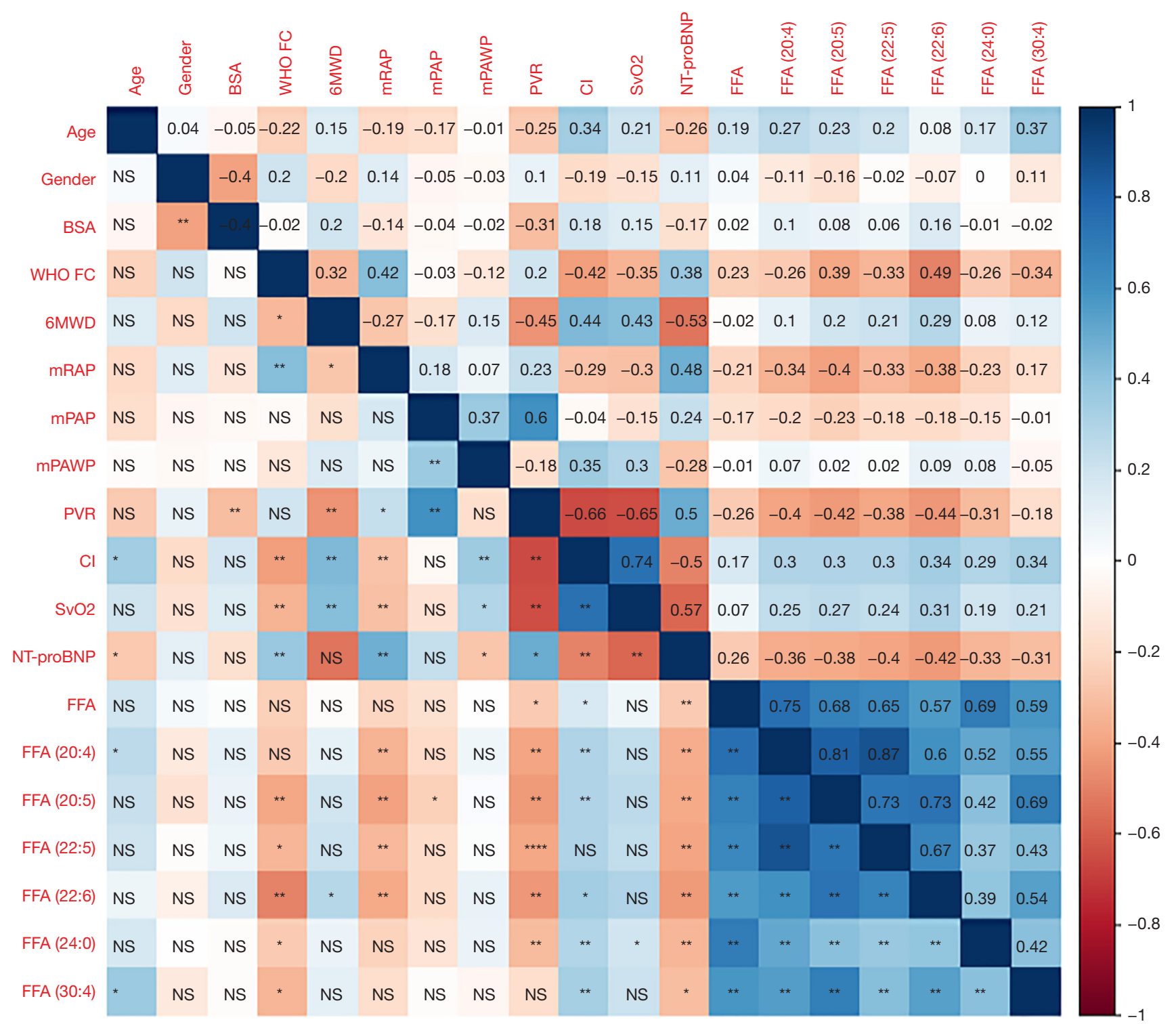

Figure 3 The relationship between lipids with significant differences and baseline characteristics in patients with IPAH. *, P $<0.05 ;$ **, $\mathrm{P}<0.01$. IPAH, idiopathic pulmonary arterial hypertension; BSA, body surface area; WHO FC, World Health Organization functional classification; 6MWD, 6-minute walking distance; mRAP, mean right atrial pressure; mPAP, mean pulmonary arterial pressure; mPAWP, mean pulmonary artery wedge pressure; PVR, pulmonary vascular resistance; CI, cardiac index; $\mathrm{SvO}_{2}$, mixed venous oxygen saturation; NTproBNP, N-terminal pro-brain natriuretic peptide; FFA, free fatty acid; NS, no significance.

Recent studies have found that cell potassium channels play an important role in pulmonary vascular smooth muscle cell over proliferation and apoptosis resistance in PAH (19). DHA may perform an anti-pulmonary hypertension role by regulating the potassium channels of pulmonary artery smooth muscle cells, so as to balance the proliferation and apoptosis of smooth muscle cells in the pulmonary artery (20). This is consistent with the results of the present study. Small lipid molecules other than EPA and DHA have been less reported in previous studies; however, because they show the same trend as EPA and DHA, they may also play a significant role in cardiovascular protection.

Our study indicated that the levels of plasma DHA were down-regulated and could predict the survival rate 


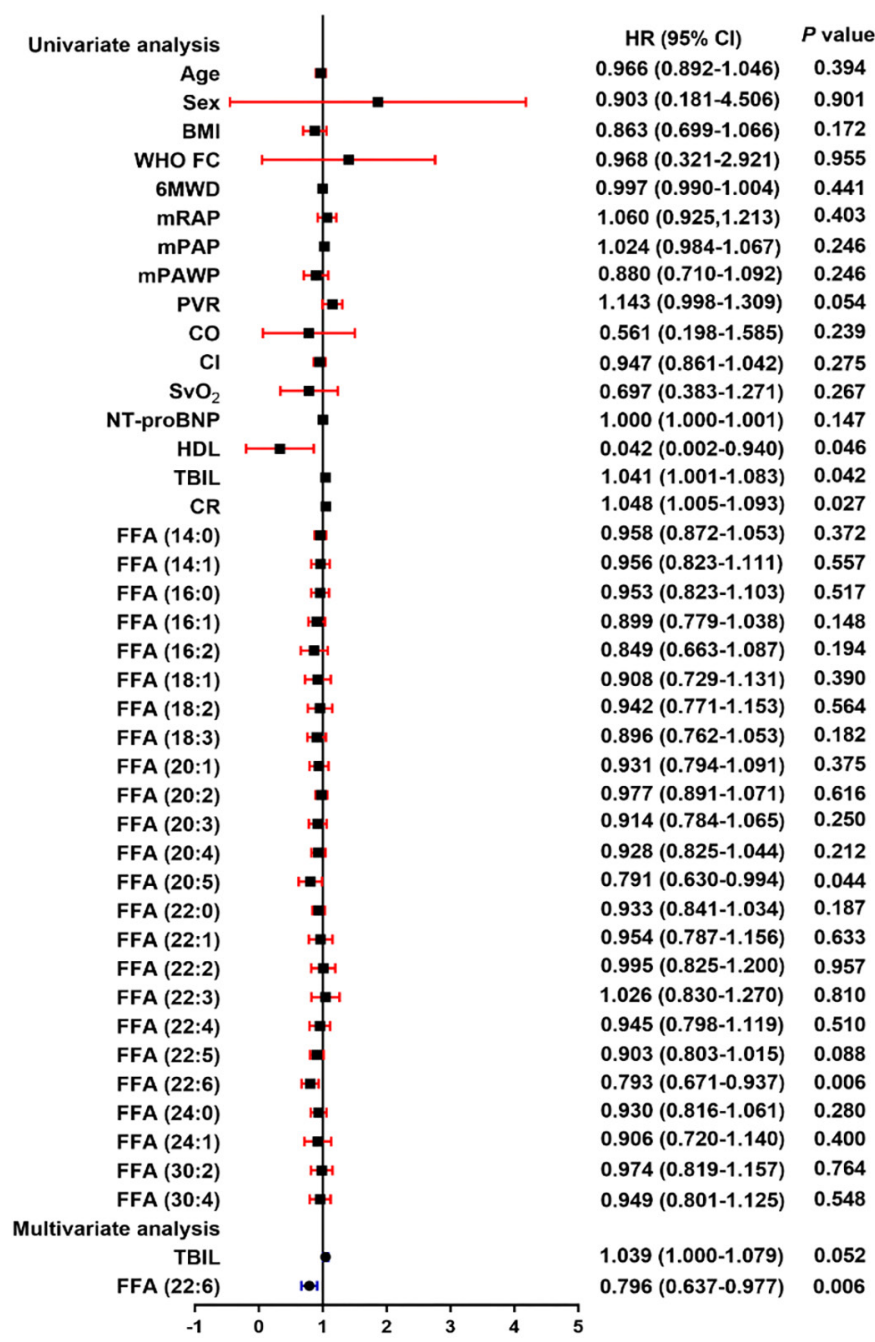

Figure 4 Forest plot showing the univariate and multivariate Cox regression analysis of IPAH. IPAH, idiopathic pulmonary arterial hypertension; BMI, body mass index; WHO FC, World Health Organization functional classification; 6MWD, 6-minute walking distance; mRAP, mean right atrial pressure; mPAP, mean pulmonary arterial pressure; mPAWP, mean pulmonary artery wedge pressure; PVR, pulmonary vascular resistance; $\mathrm{CI}$, cardiac index; $\mathrm{SvO}_{2}$, mixed venous oxygen saturation; NT-proBNP, N-terminal pro-brain natriuretic peptide; HDL, high-density lipoprotein; TBIL, total bilirubin; CR, creatinine; FFA, free fatty acid; HR, hazard ratio; 95\% CI, 95\% confidence interval.

of patients with IPAH, which lower plasma DHA may contribute to the development of IPAH. And combined with the previous studies showed that DHA is a potent vasodilator to rapidly decrease in elevated pulmonary vascular tone and pressure in pulmonary hypertension $(10,20)$. We though DHA maybe a potential therapeutic option in IPAH. Therefore, direct or indirect DHA supplementation may attenuate the progression of pulmonary arterial remodeling in patients with IPAH.
This study has several limitations. First, the studied population was small (only 69 patients), which challenges the generalizability of our results. Second, in the absence of comparisons with a healthy population, it may be biased to study lipid metabolism solely in patients with IPAH. Third, all lipid expression data in this study are relative quantity not absolute quantity, which can only judge the trend of lipid expression differences among patients with IPAH with different risk levels, and cannot obtain an authoritative 


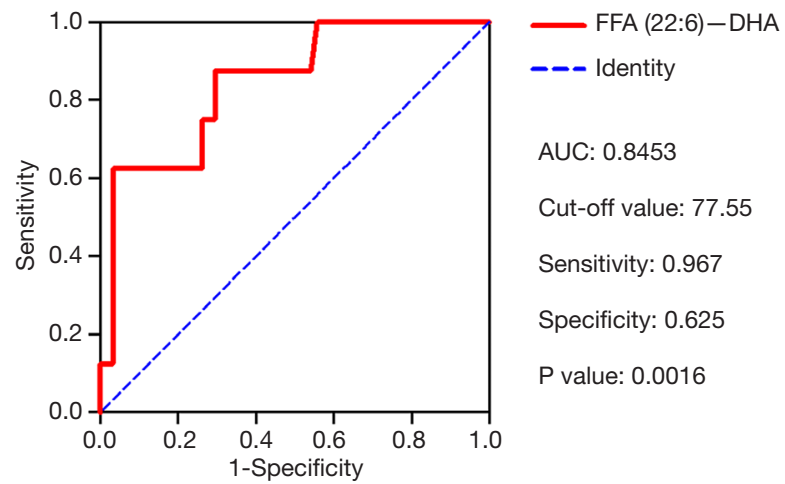

Figure 5 Receiver operating characteristic curve analysis of DHA in IPAH. DHA, docosahexaenoic acid; IPAH, idiopathic pulmonary arterial hypertension; FFA, free fatty acid; AUC, area under the curve.

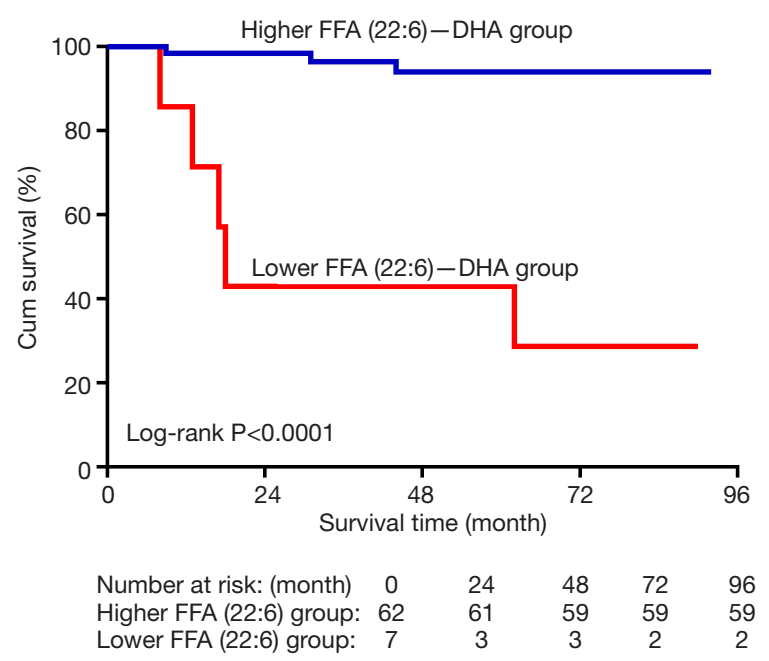

Figure 6 Predicted survival outcomes of patients with IPAH in high and low DHA groups. IPAH, idiopathic pulmonary arterial hypertension; DHA, docosahexaenoic acid; FFA, free fatty acid.

standard concentration value. Finally, the prospective study needs to further evaluate the efficacy of FFA-regulating drugs on IPAH patients.

\section{Conclusions}

FFAs were strongly associated with mortality in patients with IPAH. FFA (20:4), FFA (20:5), FFA (22:5), DHA, FFA (24:0) and FFA (30:4) have significant correlations with IPAH disease severity. A low level of DHA is predictive of poor survival in patients with IPAH. Therefore, this study may provide a theoretical basis for the treatment and daily diet of patients with IPAH in the future.

\section{Acknowledgments}

Funding: This study was supported by the Program of National Natural Science Foundation of China (81870042 and 81900050), National Science and Technology Information System of the People's Republic of China (2018YFC1313603), and Program of Natural Science Foundation of Shanghai (21ZR1453800, 18ZR1431500), and Program of Shanghai Municipal Commission of Health (20204Y0382), and Youth Project of Shanghai Municipal Commission of Health and Family Planning (20174Y0143), and Program of Shanghai Pulmonary Hospital (FKLY20005), and Program of Shanghai Pulmonary Hospital (31-20-341-027).

\section{Footnote}

Reporting Checklist: The authors have completed the STARD reporting checklist. Available at https://dx.doi. org/10.21037/atm-21-2479

Data Sharing Statement: Available at https://dx.doi. org/10.21037/atm-21-2479

Conflicts of Interest: All authors have completed the ICMJE uniform disclosure form (available at https://dx.doi. org/10.21037/atm-21-2479). The authors have no conflicts of interest to declare.

Ethical Statement: The authors are accountable for all aspects of the work in ensuring that questions related to the accuracy or integrity of any part of the work are appropriately investigated and resolved. This study complied with the Declaration of Helsinki (as revised in 2013) and was approved and supervised by the ethics committee of Shanghai Pulmonary Hospital (number: K20$195 \mathrm{Y}$ ), and written informed consent was obtained from all study participants.

Open Access Statement: This is an Open Access article distributed in accordance with the Creative Commons Attribution-NonCommercial-NoDerivs 4.0 International License (CC BY-NC-ND 4.0), which permits the noncommercial replication and distribution of the article with the strict proviso that no changes or edits are made and the 
original work is properly cited (including links to both the formal publication through the relevant DOI and the license). See: https://creativecommons.org/licenses/by-nc-nd/4.0/.

\section{References}

1. Thenappan T, Ormiston ML, Ryan JJ, et al. Pulmonary arterial hypertension: pathogenesis and clinical management. BMJ 2018;360:j5492.

2. Sardana M, Moll M, Farber HW. Novel investigational therapies for treating pulmonary arterial hypertension. Expert Opin Investig Drugs 2015;24:1571-96.

3. Guo L, Qiu Z, Wei L, et al. The microRNA-328 regulates hypoxic pulmonary hypertension by targeting at insulin growth factor 1 receptor and L-type calcium channel- $\alpha 1 \mathrm{C}$. Hypertension 2012;59:1006-13.

4. Badagliacca R, Rischard F, Papa S, et al. Clinical implications of idiopathic pulmonary arterial hypertension phenotypes defined by cluster analysis. J Heart Lung Transplant 2020;39:310-20.

5. McGoon MD, Benza RL, Escribano-Subias $\mathrm{P}$, et al. Pulmonary arterial hypertension: epidemiology and registries. J Am Coll Cardiol 2013;62:D51-9.

6. Huang C, Freter C. Lipid metabolism, apoptosis and cancer therapy. Int J Mol Sci 2015;16:924-49.

7. Kaur N, Chugh V, Gupta AK. Essential fatty acids as functional components of foods- a review. J Food Sci Technol 2014;51:2289-303.

8. Bükki J, Stanga Z, Tellez FB, et al. Omega-3 polyunsaturated fatty acids for the prevention of severe neutropenic enterocolitis in patients with acute myeloid leukemia. Nutr Cancer 2013;65:834-42.

9. Jonas K, Magoń W, Podolec P, et al. Triglyceride-tohigh-density lipoprotein cholesterol ratio and systemic inflammation in patients with idiopathic pulmonary arterial hypertension. Med Sci Monit 2019;25:746-53.

10. Nagaraj C, Tang B, Nagy BM, et al. Docosahexaenoic acid causes rapid pulmonary arterial relaxation via $\mathrm{KCa}$ channel-mediated hyperpolarisation in pulmonary hypertension. Eur Respir J 2016;48:1127-36.

Cite this article as: Huai $\mathrm{X}$, Sun $\mathrm{Y}$, Sun $\mathrm{X}, \mathrm{Wu}$ W, Wang L, Jiang R, Gong S, Li J, Miao Y, Yuan P, Zhao Q. The effect of docosahexaenoic acid on predicting the survival of patients with idiopathic pulmonary arterial hypertension. Ann Transl Med 2021;9(12):995. doi: 10.21037/atm-21-2479
11. Galiè N, Humbert M, Vachiery JL, et al. 2015 ESC/ERS Guidelines for the diagnosis and treatment of pulmonary hypertension: The Joint Task Force for the Diagnosis and Treatment of Pulmonary Hypertension of the European Society of Cardiology (ESC) and the European Respiratory Society (ERS): Endorsed by: Association for European Paediatric and Congenital Cardiology (AEPC), International Society for Heart and Lung Transplantation (ISHLT). Eur Heart J 2016;37:67-119.

12. Galiè N, Hoeper MM, Humbert M, et al. Guidelines for the diagnosis and treatment of pulmonary hypertension. Eur Respir J 2009;34:1219-63.

13. Fahy E, Cotter D, Sud M, et al. Lipid classification, structures and tools. Biochim Biophys Acta 2011;1811:637-47.

14. Hoeper MM, Kramer T, Pan Z, et al. Mortality in pulmonary arterial hypertension: prediction by the 2015 European pulmonary hypertension guidelines risk stratification model. Eur Respir J 2017;50:1700740.

15. Jickling GC, Spence JD. Free fatty acids to predict recurrent ischemic stroke. Neurology 2014;82:1110-1.

16. Spence JD. Free fatty acids and stroke from atrial fibrillation. Can J Neurol Sci 2011;38:803.

17. Seo WK, Kim J, Kim YH, et al. Elevated free fatty acid is associated with cardioembolic stroke subtype. Can J Neurol Sci 2011;38:874-9.

18. Inoguchi T, Li P, Umeda F, et al. High glucose level and free fatty acid stimulate reactive oxygen species production through protein kinase $\mathrm{C}$--dependent activation of $\mathrm{NAD}(\mathrm{P}) \mathrm{H}$ oxidase in cultured vascular cells. Diabetes 2000;49:1939-45.

19. Khawaja O, Bartz TM, Ix JH, et al. Plasma free fatty acids and risk of atrial fibrillation (from the Cardiovascular Health Study). Am J Cardiol 2012;110:212-6.

20. Karpe F, Dickmann JR, Frayn KN. Fatty acids, obesity, and insulin resistance: time for a reevaluation. Diabetes 2011;60:2441-9.

(English Language Editor: J. Reynolds) 